\title{
Determination of the recombination rate coefficients for Na-like Si Iv forming Mg-like Si III
}

\author{
I. Orban ${ }^{1}$, P. Glans ${ }^{2}$, Z. Altun ${ }^{3}$, E. Lindroth ${ }^{1}$, A. Källberg ${ }^{4}$, and R. Schuch ${ }^{1}$ \\ 1 Department of Atomic Physics, Stockholm University, 10691 Stockholm, Sweden \\ e-mail: istvan.orban@physto.se \\ 2 Department of Engineering, Physics and Mathematics, Mid Sweden University, 85170 Sundsvall, Sweden \\ 3 Department of Physics, Marmara University, 81040 Istanbul, Turkey \\ 4 Manne Siegbahn Laboratory, Stockholm University, 10405 Stockholm, Sweden
}

Received 7 December 2005 / Accepted 9 June 2006

ABSTRACT

\begin{abstract}
Aims. Absolute, total recombination rate coefficients for Si IV were determined using the CRYRING heavy-ion storage ring. Calculated rate coefficients were used to estimate recombination into states that could not be detected in the experiment because of field ionization. Total, as well as separate, radiative and dielectronic plasma recombination rate coefficients were determined. Methods. Stored ions were merged with an expanded electron beam in the electron cooler section of the storage ring. Recombined ions were separated from the stored ion beam in the first dipole magnet after the electron cooler and were detected with unity efficiency. The absolute radiative and dielectronic recombination rate coefficients were obtained over a center-of-mass energy range of $0-20 \mathrm{eV}$, covering $\Delta n=0$ core excitations up to the $3 \mathrm{~s} \rightarrow 3 \mathrm{~d}$ series limit. The results of an intermediate coupling AUTOSTRUCTURE calculation were compared with the experiment. The theoretical results were also used to estimate the contribution to dielectronic recombination by high Rydberg states, which were not detected because of field ionization. The spectra were convoluted with Maxwell-Boltzmann energy distributions in the $10^{3}-10^{6} \mathrm{~K}$ temperature range.

Results. The resulting plasma recombination rate coefficients are presented and compared with theoretical results frequently used for plasma modeling. In the $10^{3}-10^{4} \mathrm{~K}$ range, a significant underestimation of the calculated dielectronic recombination plasma rate coefficients was observed. Above $3 \times 10^{4} \mathrm{~K}$, the agreement between our dielectronic recombination plasma rate coefficients and two of the previously published rate coefficients is better than $20 \%$.

Conclusions. The observed differences between the experimental and calculated recombination rate coefficients at low temperatures reflect the need for benchmarking experiments. Our experimentally-derived rate coefficients can guide the development of better theoretical models and lead to more accurately-calculated rate coefficients.
\end{abstract}

Key words. atomic data - atomic processes - plasmas

\section{Introduction}

The absorption and emission lines of the astrophysically abundant ions, such as Si IV, CIV, N V, and O VI can be used to investigate astrophysical plasmas. With these lines it is possible to study the chemical evolution of the universe and the initial mass function of the earliest generations of stars (Savin 2000a).

Ionization-balance calculations and the correct estimations of elemental abundance in astrophysical plasmas depend on the recombination rates of the ion species present in the respective plasma. The recombination rate coefficients used for ionization balance and level population calculations originate primarily in calculations based on theoretical models. However, extrapolations to other ions in a given isoelectronic series are often not verified experimentally. This can result in a factor of 2 or more uncertainties in the different calculations for ions with partially filled L and M shells (Savin 2000b). For temperatures below $10^{4} \mathrm{~K}$, the discrepancy can be much larger, due to the inaccuracy of the calculated low-energy dielectronic recombination (DR) resonance positions and strengths. In order to obtain accurate cross sections at low energies, electron correlation up to high orders, as well as relativistic and quantum-electrodynamic effects, must be considered in calculating of the doubly excited states (Mannervik et al. 1998). The results of recombination measurements at storage rings represent the most reliable tests of such calculations.

In this paper, we present absolute recombination rate coefficients for Na-like $\mathrm{Si}$, determined from a recombination measurement at the CRYRING storage ring. The results of an intermediate coupling calculation were used to account for field ionization of high Rydberg states occurring in the dipole magnet before the detector. The obtained spectrum was then convoluted with Maxwell-Boltzmann electron energy distributions for temperatures ranging from $10^{3}-10^{6} \mathrm{~K}$. The derived temperaturedependent plasma recombination rate coefficients are presented and compared with recommended data from the literature.

One of the important processes in ion-electron collisions is radiative recombination (RR), in which an electron is captured by an ion with the simultaneous emission of a photon. This process can take place at any collision energy, and a finite probability exists for recombination to all available levels of the ion. The first expression describing the RR cross section was derived by Kramers (1923). For capture into low- $n$ states, the expression should be corrected by the Gaunt factor $g_{n}$ (Seaton 1959):

$\sigma_{n}(E)=2.105 \times 10^{-22} g_{n} \frac{\mathrm{Ry}^{2} \cdot Z_{\mathrm{eff}}^{4}}{n E\left(n^{2} E+\mathrm{Ry} \cdot Z_{\mathrm{eff}}^{2}\right)}\left[\mathrm{cm}^{2}\right]$, 
where Ry represents the reduced mass Rydberg constant, $E$ is the center-of-mass energy, $n$ the principal quantum number of the recombined electron, and $Z_{\mathrm{eff}}$ the effective charge of the ion as seen by the captured electron. The total RR cross section is obtained by summing the cross sections over all available states.

Another important recombination process is dielectronic recombination, in which an electron is typically captured to a Rydberg level $(n, l)$ while a core electron is simultaneously excited. The doubly-excited state formed is normally autoionizing; however, a small fraction of the doubly excited ions will radiatively decay and, if the energy of the formed ion becomes smaller than its first ionization energy, the recombination process is completed. Energy conservation demands that the sum of the incoming electron kinetic energy and the binding energy of the Rydberg electron after capture must equal the excitation energy of the core electron in the initial system, i.e.,

$E_{\mathrm{e}}=\Delta E_{\mathrm{ion}}-E_{\mathrm{b}}(n l)$,

where $E_{\mathrm{e}}$ is the kinetic energy of the electron, $\Delta E_{\mathrm{ion}}$ the excitation energy of the core electron in the initial system, and $E_{\mathrm{b}}$ the binding energy of the outer Rydberg electron, with respect to the excited target. This explains the resonant nature of the DR process.

\section{Experiment}

The recombination measurement was performed at the heavyion storage ring CRYRING, located at the Manne Siegbahn Laboratory in Stockholm. The Na-like Si ions were produced in an electron cyclotron resonance (ECR) ion source. Ions were injected and accelerated in the storage ring to an energy of $1.09 \mathrm{MeV} \mathrm{amu}^{-1}$. This resulted in an average of $2.3 \times 10^{6}$ ions stored after injection and acceleration.

In the electron cooler section of the storage ring, a cold electron beam was merged with the ion beam (Danared et al. 2000). During $2 \mathrm{~s}$ of electron cooling, the ion-beam phase space is reduced such that the beam was approximately $1 \mathrm{~mm}$ in diameter. The electron-accelerating potential was then varied, leading to a range of ion-electron collision energies between 0 and $20 \mathrm{eV}$ in the center-of-mass system. During the measurement, the electron current was kept constant with an electron density of $9.08 \times 10^{6} \mathrm{~cm}^{-3}$ at cooling.

The recombined ions were separated from the stored ion beam in the first dipole magnet after the electron cooler and were detected with a unity-efficiency surface-barrier detector. The motional electric field in this dipole magnet was sufficient for field-ionizing recombined ions with the outer electron in Rydberg states with $n>17$. This will be discussed in more detail in Sect. 4.2. Hereafter, the data affected by this field-ionization will be referred to as $n_{\text {cutoff }}$ rate coefficients.

The resulting count rates were transformed to merged-beam recombination rate coefficient values with a similar procedure as in DeWitt et al. (1996) and Zong et al. (1997). The obtained rate coefficients are shown as a function of the corresponding collision energy in Fig. 1.

\section{Autostructure calculation}

The DR parts of the calculated curves in Fig. 1 were obtained using the AUTOSTRUCTURE code (Badnell 1986). The calculation was performed within the multiconfiguration intermediate coupling Breit-Pauli (MCBP) approximation using non-relativistic radial functions. It provided energy levels, as well as radiative

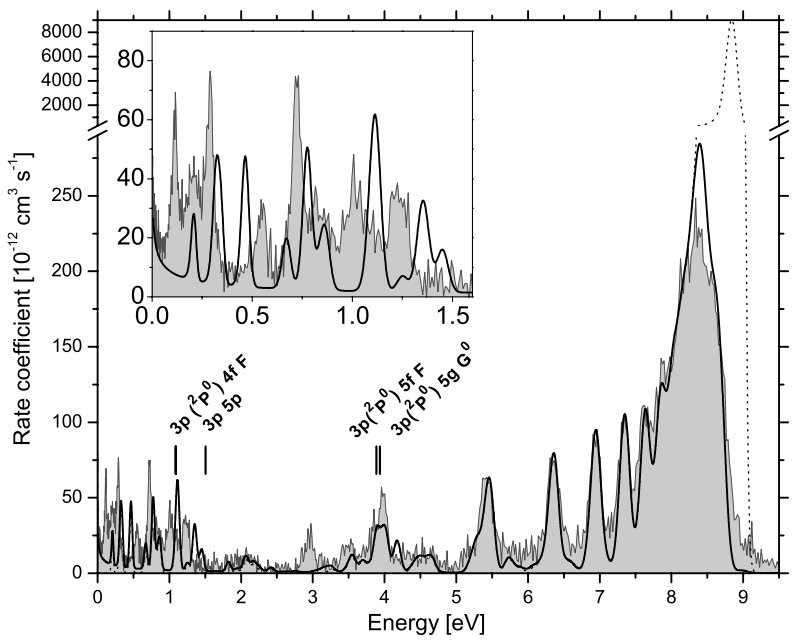

Fig. 1. The experimental spectrum is shown by the shaded area. The solid and dotted curves show calculated spectra. The solid curve accounts for field ionization in the measurement (see Sect. 2 for more details). The dotted curve includes DR resonances up to $n=1000$. The inset displays the low energy part of the spectrum in more detail. The vertical bars indicate the energy position of the doubly excited states of $\mathrm{Mg}$-like Si listed in the NIST atomic data tables (NIST database).

and autoionization rates, for the doubly excited states. The autoionization rates were calculated in the isolated resonance approximation using the distorted wave approximation. This enabled the generation of total DR rate coefficients that are level resolved (Badnell et al. 2003).

Both $\Delta n=0$ and $\Delta n=1$ core excitations have been considered in the calculation and all possible stabilizing radiative transitions were accounted for. More details about the AUTOSTRUCTURE calculation are included in Altun et al. (2006), where results for Na-like ions between $\mathrm{Mg}^{+}$and $\mathrm{Xe}^{43+}$ are presented.

\section{Results and discussion}

In the following sections, our calculated and experimentally derived recombination rate coefficients are presented and compared. Plasma recombination rate coefficients are determined and comparisons are made with data from the literature, frequently used for plasma diagnostics.

\section{1. $R R$ rate coefficients}

The RR rate coefficients shown in Fig. 2 were obtained by multiplying the calculated cross section (Eq. (1)) with the electron velocity in the center-of-mass system, and by convoluting with the electron-velocity distribution from the experiment. Motional electric fields in the experiment cause field ionization of high Rydberg states; therefore, when calculating the RR rate coefficients for comparison with the experiment, only states with $n$ up to 17 were included. The $Z_{\text {eff }}$ effective charge was determined as a function of the principal quantum number $n$, in which the electron recombines. For $n=3,4$, and 5, a weighted average was performed over the energy of the states belonging to the same shell (NIST database), taking into account the relative contribution of the levels with different orbital momenta (Pajek \& Schuch 1992). Above $n=5$ the energy of the state with the highest 


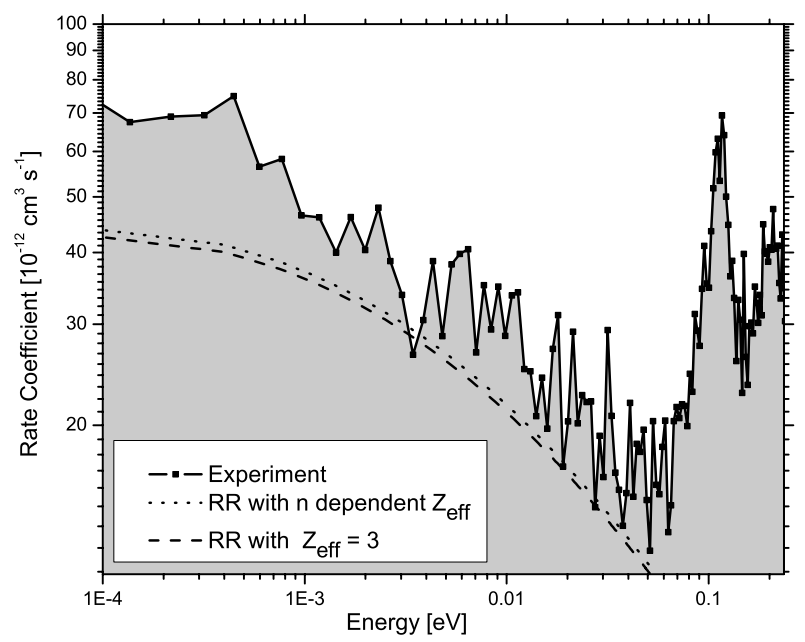

Fig. 2. Experimental results at low energies (shaded area) and the calculated RR rate coefficients obtained using the determined $n$ dependent

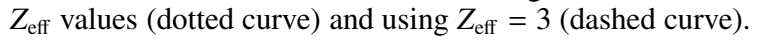

Table 1. Effective charge values obtained for the different shells in the recombined $\mathrm{Mg}$-like Si system. Above $n=9$ the effective charge was considered to be 3 .

\begin{tabular}{cccc}
\hline \hline$n$ & $Z_{\text {eff }}$ & $n$ & $Z_{\text {eff }}$ \\
\hline 3 & 3.8950 & 7 & 3.0129 \\
4 & 3.4210 & 8 & 3.0125 \\
5 & 3.2416 & 9 & 3.0036 \\
6 & 3.0132 & $>9$ & 3 \\
\hline
\end{tabular}

contributing orbital momentum was taken. The resulting energies were used in the hydrogenic formula

$E(n)=Z_{\mathrm{eff}}^{2} \frac{\mathrm{Ry}}{n^{2}}$,

to extract the value of the effective charge corresponding to each $n$. In Eq. (3), $E(n)$ is the averaged energy for shell $n$, and $\mathrm{Ry}=13.6057 \mathrm{eV}$ is the binding energy of the hydrogen atom. The obtained effective charge values are given in Table 1 .

The RR rate coefficients calculated using the obtained effective charge values are presented in Fig. 2 and were also used for the RR part of the calculated curves in Fig. 1. The discrepancy with the experiment in the $10^{-3}-5 \times 10^{-2} \mathrm{eV}$ range is mainly caused by low-energy DR resonances that contribute to the measured recombination rate coefficient at low energies, because of the $E^{-1}$ dependence of the DR cross section. Adding the contribution of the low-energy DR resonances to the calculated RR, a good agreement with experimental data is obtained. More details about this can be found in Orban et al. (2006).

Below $1 \mathrm{meV}$, the experimental recombination rate is clearly enhanced compared to the rate given by the Kramers formula. This effect is a common characteristic of storage ring experiments (Gao et al. 1995), and its origin is not completely clear yet.

\subsection{DR rate coefficients}

The DR peaks presented in Fig. 1 are due to doubly excited states in $\mathrm{Mg}$-like $\mathrm{Si}$. The following $\Delta n=0 \mathrm{DR}$ transitions are possible:

$\mathrm{Si}^{3+}(3 \mathrm{~s})+\mathrm{e}^{-}(\varepsilon l) \leftrightarrow \mathrm{Si}^{2+}(3 \mathrm{p}, n l) \rightarrow\left\{\begin{array}{l}\mathrm{Si}^{2+}(3 \mathrm{~s}, n l)+h v \\ \mathrm{Si}^{2+}\left(3 \mathrm{p}, n^{\prime} l^{\prime}\right)+h v^{\prime},\end{array}\right.$ and

$\mathrm{Si}^{3+}(3 \mathrm{~s})+\mathrm{e}^{-}(\varepsilon l) \leftrightarrow \mathrm{Si}^{2+}(3 \mathrm{~d}, n l) \rightarrow\left\{\begin{array}{l}\mathrm{Si}^{2+}(3 \mathrm{p}, n l)+h v \\ \mathrm{Si}^{2+}\left(3 \mathrm{~d}, n^{\prime} l^{\prime}\right)+h v^{\prime},\end{array}\right.$

The $(3 \mathrm{p}, n l)$ doubly-excited states give rise to resonances at energies below the $3 \mathrm{p}_{1 / 2}$ and $3 \mathrm{p}_{3 / 2}$ series limits situated at about $8.9 \mathrm{eV}$. The two $3 \mathrm{p}$ series limits are separated by only $57.2 \mathrm{meV}$ (NIST database) and could not be resolved in the experimental spectrum. No resonances were observed above these series limits. Thus, all the observed $(3 \mathrm{~d}, n l)$ resonances also appear below the $3 p$ series limits.

Recombined ions with the outer electron in $n>n_{\text {cutoff }}=17$ were re-ionized by the motional electric field in the dipole magnet (Fogle et al. 2003) and remained undetected, causing a truncation in the experimental spectrum at the series limit. However, the cutoff in the spectrum is not sharp, because during the $124 \mathrm{~ns}$ flight time between the electron cooler and the dipole magnet, some of these ions radiatively decayed to states with the outer electron in a level with $n \leq n_{\text {cutoff }}$. These recombined ions preserved their charge state while passing through the dipole magnet and were recorded by our detector.

The energy positions from the AUTOSTRUCTURE calculation are accurate for the $(3 \mathrm{p}, n l)$ states with high $n$, because of the small correlation between the two electrons. In order to align the high-energy peaks of the experiment with the AUTOSTRUCTURE results, the experimental energies had to be multiplied with 1.007, which is well within the estimated error of up to a few percent for this type of experiment (Lindroth \& Schuch 2003). The error in the experimental rate coefficient is estimated to be around $15 \%$ and originates mainly from uncertainties in the ion current, the ion beam orbit length, the length of the electron-ion interaction region, and statistical errors.

Between $5 \mathrm{eV}$ and $8.2 \mathrm{eV}$, as seen in Fig. 1, the overall agreement between the calculation up to $n_{\text {cutoff }}$ and experiment is good. In the region close to the field ionization limit, the experimentally-determined rate coefficient curve is slightly lower than the AUTOSTRUCTURE data. The lower rate in the experiment near $n_{\text {cutoff }}$ might be due to reduction in the strength of some of the $(3 \mathrm{p}, n l)$ resonances with $n$ equal to, or slightly smaller than, $n_{\text {cutoff }}$, due to partial field ionization in the dipole magnet. Below $5 \mathrm{eV}$ the agreement between the calculated and experimental spectrum is poor. The most prominent difference between the two spectra is the absence in the AUTOSTRUCTURE spectrum of the peak at $2.97 \mathrm{eV}$, which is due to the $\left(3 \mathrm{~d}^{2}\right)^{3} F$ doubly-excited states (Orban et al. 2006). The autoionization of these doubly excited states are nonrelativistically forbidden which, together with the strong electron correlation for these states, makes accurate calculation of the position and strength of these resonances very difficult.

The inset in Fig. 1 shows the low-energy DR peaks, and the discrepancy between the two spectra indicates the need to fully account for electron correlation in the calculation of doubly excited states. Although the number of peaks is almost the same as in the experiment, the resonance strengths and positions are not reproduced correctly by the calculation; the peaks are located at higher energies, and the recombination rate coefficients are lower than the corresponding experimental values.

The DR resonances in Fig. 1 are mainly due to the $(3 \mathrm{p}, n l)$ doubly excited states. The overlapping resonances located between $3.0 \mathrm{eV}$ and $4.7 \mathrm{eV}$ correspond mainly to the (3p, $5 l)$ doubly excited states. The peak due to the $(3 \mathrm{p}, 6 l)$ doubly excited states is located at about $5.45 \mathrm{eV}$ and the resonances centered at $6.35 \mathrm{eV}$ correspond mainly to the $(3 \mathrm{p}, 7 l)$ states. Resonances centered at $6.9 \mathrm{eV}$ are due to $(3 \mathrm{p}, 8 l)$ doubly excited states. Some 
of the lower members of the $(3 \mathrm{p}, 9 l)$ resonances overlap with $(3 \mathrm{p}, 8 l)$ resonances; the contribution from the $(3 \mathrm{p}, 9 l)$ states has a maximum at $7.34 \mathrm{eV}$.

Figure 1 also shows the results of the AUTOSTRUCTURE calculation for recombination into states with $\mathrm{n}$ up to 1000 . Selecting $n=1000$ is a good approximation for infinity, i.e., the case when none of the recombined states are field-ionized in the dipole magnet. Hereafter the recombination data up to this quantum number are referred to as field-free rate coefficients. In Fig. 1, the $n_{\text {cutoff }}$ and field-free AUTOSTRUCTURE data have the same values below $8.2 \mathrm{eV}$.

\subsection{Temperature-dependent plasma recombination rate coefficients}

The merged-beam recombination rate coefficients discussed so far are given as a function of the ion-electron collision energies in the center-of-mass system and they show a detailed spectrum with many individual DR resonances. The plasma recombination rate coefficients, on the other hand, are expressed as a function of plasma temperature, and they include a contribution from all recombination channels, weighted by the energy distribution of the electrons at the respective temperature. The plasma recombination rate coefficient $\alpha\left(T_{\mathrm{e}}\right)$ was obtained by convoluting the recombination spectra from Fig. 1 with the Maxwell-Boltzmann energy distribution of the electrons in a plasma at a temperature $T_{\mathrm{e}}$

$\alpha\left(T_{\mathrm{e}}\right)=\int \alpha(E) f\left(E, T_{\mathrm{e}}\right) \mathrm{d} E$,

where $\alpha(E)$ is the merged-beam recombination rate coefficient, and $f\left(E, T_{\mathrm{e}}\right)$ is the Maxwell-Boltzmann distribution of the electron energies, as given by

$f\left(E, T_{\mathrm{e}}\right)=\frac{2 E^{1 / 2}}{\pi^{1 / 2}\left(k T_{\mathrm{e}}\right)^{3 / 2}} \exp \left(-\frac{E}{k T_{\mathrm{e}}}\right)$.

Equation (6) is valid as long as the merged-beam recombination rate coefficient spectrum is a good approximation of the recombination cross section, i.e., as long as the experimental electron temperatures are much lower than the temperature of the Maxwell-Boltzmann energy distributions (Schippers et al. 2001). The effect of the experimental resolution on our DR plasma rate coefficients was found to be a relative difference of $<4 \%$ between $10^{3}$ and $2.5 \times 10^{3} \mathrm{~K}$ and $<1 \%$ above this range.

The convolution of the measured recombination rate coefficient spectrum was made over a temperature range of $10^{3}-10^{6} \mathrm{~K}$. The derived total $(\mathrm{RR}+\mathrm{DR}) n_{\text {cutoff }}$ plasma rate coefficients are shown in Fig. 3.

The merged-beam RR rate coefficients calculated with $n$ dependent $Z_{\text {eff }}$ (see Sect. 4.1) were used to obtain the RR plasma rate coefficients. The same RR rate coefficients were also used to subtract the RR contribution from the measured merged-beam recombination spectrum. The resulting experimental DR spectrum was then convoluted with Maxwell-Boltzmann energy distributions. The derived $n_{\text {cutoff }}$ DR plasma rate coefficients are shown in Fig. 3.

To obtain field-free DR plasma rate coefficients, the experimental DR spectrum from Fig. 1 was replaced above $8.2 \mathrm{eV}$ by the field-free AUTOSTRUCTURE data. The resulting DR rate coefficients were convoluted and the derived field-free DR plasma rate coefficients are shown in Fig. 3. The total field-free plasma rate coefficient was obtained by adding together the field-free RR and field-free DR plasma rate coefficients. At low and at

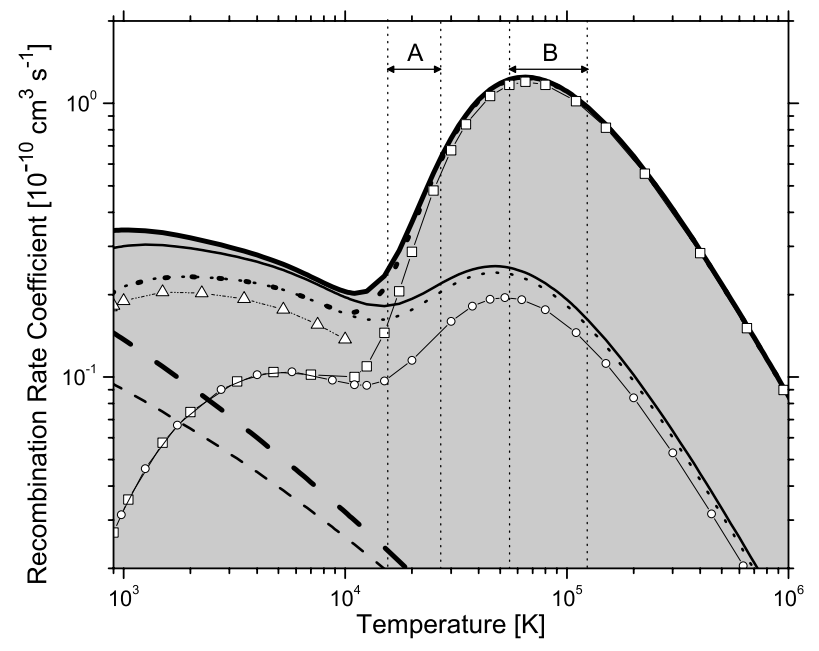

Fig. 3. Plasma recombination rate coefficients for Si IV as a function of temperature. The thin and thick dashed lines show the calculated $n_{\text {cutoff }}$ and field-free RR rate coefficients, respectively. The thin-dotted and thick-dotted curves present the experimentally-derived $n_{\text {cutoff }}$ and field-free DR recombination rate coefficients, respectively (see Sect. 4.3 for more details). The total ( $\mathrm{RR}+\mathrm{DR}$ ) rate coefficients for the $n_{\text {cutoff }}$ and field-free conditions are shown by thin and thick solid curves, respectively. The $n_{\text {cutoff }}$ and field-free DR plasma rate coefficients, derived using the results of the AUTOSTRUCTURE calculation, are shown by open circles and open squares, respectively. The plasma rate coefficients derived from the combination of a relativistic MBPT calculation up to $1.8 \mathrm{eV}$ with a nonrelativistic MBPT calculation up to $6.7 \mathrm{eV}$ (Orban et al. 2006), are shown by open triangles. The temperature ranges, for which the Si IV abundance is higher than $90 \%$ of the maximum occurrence in photoionized and collisionally ionized plasmas, are shown as regions labeled A and B, respectively (Kallman \& Bautista 2001).

high temperatures, a difference appears between the $n_{\text {cutoff }}$ total plasma rate coefficient and the field-free total plasma rate coefficient curves. The difference around $\sim 10^{3} \mathrm{~K}$ appears because of the field-ionization of states with $n>n_{\text {cutoff }}$, populated via RR.

At higher temperatures, DR is the dominant recombination process. In Fig. 3 , the $n_{\text {cutoff }}$ DR and field-free DR plasma rate coefficients have the same values up to $10^{4} \mathrm{~K}$; above this temperature high Rydberg states, for which field ionization plays a role, begin to contribute significantly. The contribution from these high Rydberg states also causes the difference at high temperatures between the $n_{\text {cutoff }}$ and field-free total rate coefficients.

The pure AUTOSTRUCTURE DR plasma rate coefficients for the $n_{\text {cutoff }}$ and field-free cases are shown for comparison. The discussed discrepancies between the calculated and the experimental spectra at low energies (Fig. 1) cause the lower AUTOSTRUCTURE plasma rate coefficients at low temperatures. The combined results of one relativistic and one nonrelativistic many body perturbation theory (MBPT) calculation (Tokman et al. 2002; Orban et al. 2006) are shown in Fig. 3. These calculations include only resonances up to $6.7 \mathrm{eV}$ and the plasma rate coefficients are therefore only shown for low temperatures, up to $10^{4} \mathrm{~K}$. In this temperature range the MBPT data are closest to the experimentally-determined DR plasma rate coefficients.

The experimental and field-free DR plasma rate coefficient curves were fitted using:

$\alpha\left(T_{\mathrm{e}}\right)=T_{\mathrm{e}}^{-3 / 2} \sum_{i} c_{i} \cdot \exp \left(-\frac{E_{i}}{k_{\mathrm{B}} T_{\mathrm{e}}}\right)$,

where $c_{\mathrm{i}}$ and $E_{\mathrm{i}}$ are fit parameters. This equation has the same $T_{\mathrm{e}}^{-3 / 2}$ electron plasma temperature dependence as the formula 


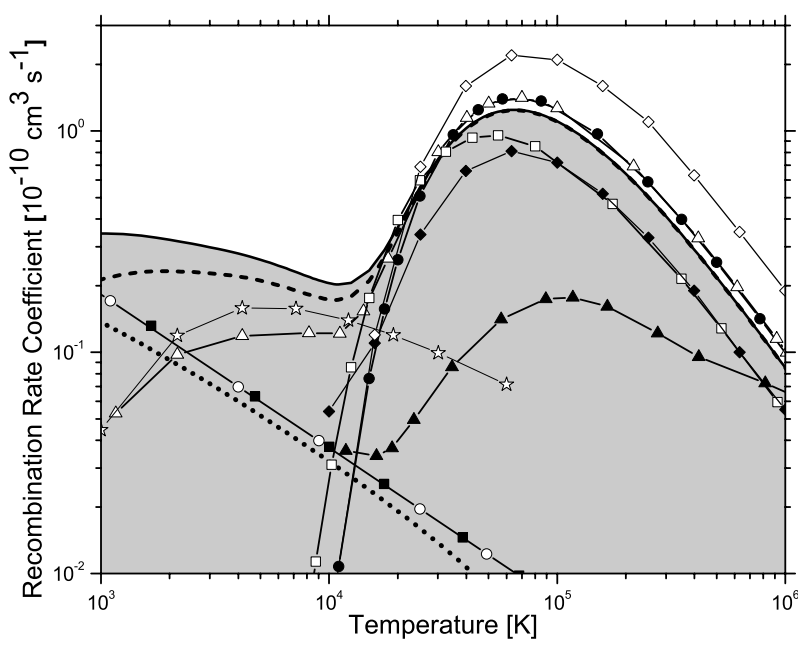

Fig. 4. The shaded area shows the experimentally-derived total plasma rate coefficient, and represents the sum of the field-free RR (dots) and field-free DR (dashed line) rate coefficients. The RR and DR rate coefficients of Aldrovandi \& Pequignot (1973) are shown as open and full circles, respectively. The full and open squares show the RR and DR rate coefficients of Mewe et al. (1980), respectively. The full triangles present the data of Hulse (1983), taken from Kato \& Asano (1999), and the open triangles show the DR recombination coefficients of $\mathrm{Gu}$ (2004). The DR rate coefficients calculated by Nussbaumer \& Storey (1986) are shown by stars. The full diamonds show the DR results of Jacobs et al. (1977), and the open diamonds present the DR rate coefficients from the same reference, calculated with the formula given by Burgess (1965; see text).

Table 2. Fitting parameters for the field-free and experimental DR plasma rate coefficients presented in Fig. 3. The parameters were obtained using Eq. (8). The dimensions of $c_{i}$ and $E_{i}$ are $10^{-5} \mathrm{~cm}^{3} \mathrm{~s}^{-1} \mathrm{~K}^{1.5}$ and $\mathrm{eV}$, respectively.

\begin{tabular}{ccccc}
\hline \hline \multicolumn{3}{c}{ Experimental DR } & \multicolumn{2}{c}{ Field-free DR } \\
$i$ & $c_{i}$ & $E_{i}$ & $c_{i}$ & $E_{i}$ \\
\hline 1 & 0.26 & 0.126 & 0.278 & 0.13 \\
2 & 0.83 & 0.407 & 0.901 & 0.43 \\
3 & 3.0 & 1.077 & 3.0 & 1.16 \\
4 & 13 & 3.86 & 18 & 4.24 \\
5 & 114 & 7.87 & 916 & 8.71 \\
\hline
\end{tabular}

given by Burgess (1965). The resulting fit parameters are shown in Table 2 and reproduce the rate coefficient curves in the presented temperature range to within $0.6 \%$.

In Fig. 4, the derived field-free plasma recombination rate coefficients are compared with recombination data from the literature, given by commonly-used formulas for astrophysical and laboratory plasmas. The RR plasma rate coefficients of Aldrovandi \& Pequignot (1973) and Mewe et al. (1980) agree within the presented temperature range. These rate coefficients are higher than our calculated field-free RR plasma rate coefficients.

Above $4 \times 10^{4} \mathrm{~K}$, the DR plasma rate coefficients of Aldrovandi \& Pequignot (1973) agree with the DR results of $\mathrm{Gu}$ (2004). Their data are larger than our field-free DR plasma rate coefficients by $12 \%$ at $4 \times 10^{4} \mathrm{~K}$ and by $20 \%$ at $10^{6} \mathrm{~K}$. In the same temperature range, the DR data of Mewe et al. (1980) is lower than our plasma rate coefficients by $10.5 \%$ and $37 \%$ at $4 \times 10^{4} \mathrm{~K}$ and $10^{6} \mathrm{~K}$, respectively.

Above $2 \times 10^{4} \mathrm{~K}$, the DR plasma rate coefficient curve by Jacobs et al. (1977) has a similar shape to our DR data. Their rate coefficients have lower values than our results by $69 \%$ at $10^{4} \mathrm{~K}$ and $\sim 35 \%$ above $4 \times 10^{4} \mathrm{~K}$. These DR results were fitted by Shull \& Steenberg (1982); however, at $6 \times 10^{4} \mathrm{~K}$ the rate coefficients, calculated with the fit coefficients presented in their publication, are $\sim 85$ times larger than the original data by Jacobs et al. (1977) for this ion. Another set of DR plasma rate coefficients from Jacobs et al. (1977), calculated with the formula given by Burgess (1965), are also shown. Above $3 \times 10^{4} \mathrm{~K}$ these rate coefficients are substantially larger than our field-free DR data.

The most dramatic difference is observed in the case of the total plasma recombination rate coefficients of Hulse (1983), taken from Kato \& Asano (1999). These rate coefficients are calculated using data from the atomic transport code MIST by Hulse (1983), which is often used for modeling magnetically confined plasmas. These results are almost an order of magnitude lower than our data at $10^{5} \mathrm{~K}$ and have comparable values to our field-free RR plasma rate coefficients at $10^{4} \mathrm{~K}$.

For temperatures below $10^{4} \mathrm{~K}$, our DR plasma rate coefficients are not influenced by the $n_{\text {cutoff }}$ value; i.e., the plasma recombination rate coefficient is almost entirely due to resonances located below $n_{\text {cutoff }}$. In this temperature range, the contribution of the AUTOSTRUCTURE data, used to extend the experimental recombination spectrum to the field-free case (see Sect. 4.2), is negligible. Below $10^{4} \mathrm{~K}$, the DR results of Aldrovandi \& Pequignot (1973) and Mewe et al. (1980) are significantly different from our DR rate coefficients.

The DR plasma rate coefficients, calculated from coefficients given by $\mathrm{Gu}$ (2004), are plotted for temperatures down to $1.16 \times 10^{3} \mathrm{~K}$. When compared to our DR data, substantial underestimation of the DR rate coefficients below $1.5 \times 10^{4} \mathrm{~K}$ can be observed. This discrepancy can be explained by the uncertainties in the low-energy resonance positions used in the calculation of $\mathrm{Gu}$ (2004), as pointed out by the author. He expects the calculation to be unreliable below the presented temperature range.

Nussbaumer \& Storey (1986) present DR plasma rate coefficients for the $10^{3}-6 \times 10^{4} \mathrm{~K}$ range. Their calculation is done in LS coupling, using a weak interaction approximation, which allows the autoionization of all states above the first ionization threshold. Below $1.3 \times 10^{4} \mathrm{~K}$, the DR results of Nussbaumer \& Storey (1986) are higher than the rate coefficients of Gu (2004) and are closer to our data. However, toward lower temperatures the difference from our field-free data increases significantly, and at $10^{3} \mathrm{~K}$ the data of Nussbaumer \& Storey (1986) have comparable values to the results of $\mathrm{Gu}(2004)$. Above $1.3 \times 10^{4} \mathrm{~K}$, Nussbaumer \& Storey (1986) underestimate our recommended DR plasma rate coefficient. At $6 \times 10^{4} \mathrm{~K}$, they have values more than one order of magnitude lower than our plasma rate coefficients. The lower rate coefficients above $1.3 \times 10^{4} \mathrm{~K}$ are probably due to DR into levels with $n>7$, which were not included in the calculation of Nussbaumer \& Storey (1986).

\section{Conclusions}

The Si IV temperature-dependent RR and DR plasma rate coefficients were determined from a recombination measurement at the CRYRING storage ring. In the measurement, the DR resonances up to $20 \mathrm{eV}$ were investigated. Here we have presented the spectrum of doubly excited states up to the $(3 \mathrm{p}, n l)$ series limits. Above the energy corresponding to the $(3 \mathrm{p}, n l)$ series limits, no DR resonances were observed.

The result of an AUTOSTRUCTURE calculation and the experimental data are in good agreement above $5 \mathrm{eV}$. For lower energies, significant discrepancies can be observed. The 
AUTOSTRUCTURE calculation was used to compensate the measured recombination spectrum for the field ionization occurring in the dipole magnet. The resulting field-free and the $n_{\text {cutoff }}$ experimental rate coefficients were both convoluted with MaxwellBoltzmann electron-energy distributions over the $10^{3}-10^{6} \mathrm{~K}$ temperature range in order to determine the plasma recombination rate coefficients. Comparisons were made with the recombination data widely used in astrophysical calculations, and a general underestimation of the DR rate coefficients was observed for temperatures below $1.5 \times 10^{4} \mathrm{~K}$. For these temperatures only the results of Gu (2004) and Nussbaumer \& Storey (1986) show significant DR rate coefficients.

At $6 \times 10^{4} \mathrm{~K}$, different calculations yield DR plasma rate coefficients that differ by as much as a factor of 30 . Above this temperature, the DR plasma rate coefficients by Aldrovandi \& Pequignot (1973) and the DR results of Gu (2004) are closest to our data.

Acknowledgements. We are grateful to Prof. N. Badnell, Prof. D. Savin, S. Böhm, and M. Fogle for fruitful discussions. We would like to thank the CRYRING staff members at Manne Siegbahn Laboratory, especially A. Simonsson, A. Paál, and M. Blom, for assistance during the experiment.

\section{References}

Aldrovandi, S. M. V., \& Pequignot, D. 1973, A\&A, 25, 137

Altun, Z., Yumak, A., Badnell, N. R., et al. 2006, A\&A, 447, 1165
Badnell, N. R. 1986, J. Phys. B, 19, 3827

Badnell, N. R., O’Mullane, M., Summers, H. P., et al. 2003, A\&A, 406, 1151

Burgess, A. 1965, ApJ, 141, 1588

Danared, H., Källberg, A., Andler, G., et al. 2000, Nucl. Instrum. Meth. A, 441, 123

DeWitt, D. R., Schuch, R., Gao, H., et al. 1996, Phys. Rev. A, 53, 2327

Fogle, M., Badnell, N. R., Eklöw, N., et al. 2003, A\&A, 409, 781

Gao, H., DeWitt, D. R., Schuch, R., et al. 1995, Phys. Rev. Lett., 75, 4381

Gu, M. F. 2004, ApJS, 153, 389

Hulse, R. A. 1983, Nuclear Technology/Fusion, 3, 259

Jacobs, V. L., Davis, J., Kepple, P. C., \& Blaha, M. 1977, ApJ, 215, 690

Kallman, T., \& Bautista, M. 2001, ApJS, 133, 221

Kato, T., \& Asano, E. 1999, NIFS-DATA-54

Kramers, H. A. 1923, Philos. Mag., 46, 836

Lindroth, E., \& Schuch, R. 2003, Recombination of cooled highly charged ions with low-energy electrons, in The Physics of Multiply and Highly Charged Ions, ed. F. J. Currell (Kluwert Academic Publishers)

Mannervik, S., DeWitt, D., Engström, L., et al. 1998, Phys. Rev. Lett., 81, 313

Mewe, R., Schrijver, J., \& Sylwester, J. 1979, A\&AS, 40, 323

NIST database:

http://physics.nist.gov/PhysRefData/ASD/index.html

Nussbaumer, H., \& Storey, P. J. 1986, A\&AS, 64, 545

Orban, I., Lindroth, E., Glans, P., et al. 2006, in preparation

Pajek, M., \& Schuch, R. 1992, Phys. Rev. A, 45, 11, 7894

Savin, D. W. 2000a, ApJ, 533, 106

Savin, D. W. 2000b, Rev. Mex. Astron. Astrofis., 9, 115

Schippers, S., Müller, A., Gwinner, G., et al. 2001, ApJ, 555, 1027

Seaton, M. J. 1959, MNRAS, 119, 81

Shull, J. M., \& Van Steenberg, M. 1982, ApJS, 48, 95

Tokman, M., Eklöw, N., Glans, P., et al. 2002, Phys. Rev. A, 66, 012703

Zong, W., Schuch, R., Lindroth, E., et al. 1997, Phys. Rev. A, 56, 386 